\title{
Alterations in Baseline Activity and Quinpirole Sensitivity in Putative Dopamine Neurons in the Substantia Nigra and Ventral Tegmental Area after Withdrawal from Cocaine Pretreatment
}

Wei-Ying Gao, M.S., Tong H. Lee, M.D., Ph.D., George R. King, Ph.D., and Everett H. Ellinwood, M.D.

Using in vivo single-unit recording, we compared in rats the effects of continuous infusion and once-a-day injections of cocaine on the activity of single putative dopamine neurons in the substantia nigra and ventral tegmental area. After a 7-day withdrawal, we determined: (1) the number of spontaneously active neurons and their bursting patterns and (2) sensitivity of these neurons to intravenous quinpirole. In the substantia nigra, continuous cocaine infusion reduced the number of neurons without affecting the bursting patterns; daily injections were without effects. In the ventral tegmental area, continuous infusion reduced the bursting activity without affecting the number of neurons, whereas injections increased number of neurons without changes in the bursting pattern. Acute sulpiride normalized all the changes in both cell body areas. The quinpirole sensitivity was selectively increased in the nigral neurons following withdrawal from continuous infusion. Possible role of $\mathrm{D}_{2}$ /autoreceptor mechanisms in these changes is discussed. [Neuropsychopharmacology 18:222-232, 1998] (C) 1998 American College of Neuropsychopharmacology. Published by Elsevier Science Inc.
KEY WORDS: Rats; Single-Unit recording; Dopamine; Cocaine; Spontaneous activity; Burst pattern; Quinpirole

In animals, chronic intermittent cocaine or amphetamine injections produce sensitization to their ability to stimulate locomotor activity and stereotypy. This behavioral phenomenon has been hypothesized as a model of the increasing reinforcing effects of cocaine in humans, especially during the early stages of abuse

From the Department of Psychiatry, Duke University Medical Center, Durham, North Carolina.

Address correspondence to: Tong H. l_ee, M.D., Ph.D., DUMC 3870, Duke University Medical center, Durham NC 27710.

Received April 28, 1997; revised July 1, 1997; accepted August 27, 1997.
(Kilbey and Ellinwood 1977; Post and Contel 1983; Kalivas 1995). In contrast to intermittent injections, continuous stimulant infusion (Ellinwood and Lee 1983; Lee and Ellinwood 1989; Reith et al. 1987; King et al. 1992) or other sustained dosing regimens (Emmett-Oglesby et al. 1993; Weiss et al. 1995) induce tolerance to the motor or reinforcing effects of the drugs. We and others have proposed that tolerance-inducing pretreatment regimens may provide an animal model for elucidating time-dependent alterations associated with stimulant withdrawal after high-dose, compulsive binges (Gawin and Ellinwood 1988; Weiss et al. 1995).

Among various electrophysiological changes, the two dosing regimens are associated with distinctive changes in the potency of dopamine (DA) agonists in 
inhibiting activity of single DA neuroris in the substantia nigra zona compacta (SNC) and ventral tegmental area (VTA). For example, the reduced apomorphine potency in the SNC during an early withdrawal (first 1-3 days) from intermittent injections (Kamata and Rebec 1984; Pitts et al. 1989, 1993; Lee et al. 1997) may be due primarily to changes in a $D_{1}$-dependent mechanism (i.e., reversed by $\mathrm{D}_{1}$ antagonist, SCH 23390; Pitts et al. 1989, 1993, Lee et al. 1997), whereas that associated with continuous infusion (Ellinwood and Lee 1983; Lee and Ellinwood 1989; Zhang et al. 1992) is mediated by a direct change in $\mathrm{D}_{2}$ receptor (e.g., autoreceptor) sensitivity (Lee et al. 1997). The findings in the VTA, on the other hand, are most consistent with autoreceptor subsensitivity (Henry et al. 1989; Lee et al. 1997; White et al. 1995) and normosensitivity (Lee et al. 1997) of soma/ dendritic autoreceptors in the early withdrawal from intermittent and continuous regimens, respectively.

After a longer withdrawal period (e.g., 7 days), intermittent injections generally lead to normosensitivity of SNC and VTA DA neurons to direct DA agonists (Henry et al., 1989; White et al. 1995). Continuous infusion, in contrast, is associated with supersensitivity of SNC DA neurons to either apomorphine in vivo (Ellinwood and Lee 1983; Lee and Ellinwood 1989; Zhang et al.. 1992) or DA in vitro (Lee et al. 1993). We have proposed that this sensitivity increase in the continuous pretreatment group may represent soma/dendritic DA autoreceptor supersensitivity.

Among various changes, altered soma/dendritic DA autoreceptor sensitivity may be manifested by changes in the number of spontaneously active DA neurons encountered during multiple passing of electrodes through the SNC or VTA (i.e., population sampling). For example, the VTA autoreceptor subsensitivity after a short withdrawal from intermittent stimulant injections has been associated with a significant increase in the number of spontaneously active DA neurons in this cell body area (Henry et al. 1989; White et al. 1995). An autoreceptor supersensitivity may conversely lead to a corresponding decrease in the number of active neurons under baseline conditions (Bunney and Grace 1978; Gallager et al. 1978).

In addition to the number of spontaneously active DA neurons, overall baseline DA neurotransmission may be determined by changes in the bursting pattern of neuronal activity (Gonon 1988). Therefore, alterations in this index after withdrawal from chronic cocaine pretreatment may have substantial effects on the functional status of DA neurotransmission in the terminal areas such as the caudate nucleus and nucleus accumbens.

In the present study, we have examined, after 7-day withdrawal, the effects of intermittent and continuous cocaine pretreatment on the baseline activity of SNC and VTA neurons exhibiting the electrophysiological characteristics of DA neurons (Grace and Bunney 1984; henceforth, we refer to these neurons as "putative DA neurons" or "DA neurons" while acknowledging that unequivocal identification of each neuron as such was not possible in this study). In addition to the population sampling, the sensitivity of these neurons to systemic quinpirole, a direct $\mathrm{D}_{2}$ agonist, was measured. Considering the striking dependence of functional outcomes on the pretreatment regimen, one of the main aims of this study was to compare and contrast the effects of the two regimens under identical experimental conditions. We now report that the different regimens lead to distinctive sets of changes in the spontaneous baseline activity and quinpirole sensitivity of single putative DA neurons 7 days after withdrawal.

\section{METHODS}

\section{Animals and Pretreatment}

Male Sprague-Dawley rats (Charles-River, Raleigh, NC), weighing 300-350 $\mathrm{g}$ at the time of recording, were used throughout the experiment. They were housed two per cage under a 12/12-h light/dark cycle. Food and water were available ad libitum. After a minimum of 7 days of acclimatization, rats were pretreated for 14 days with one of the three pretreatment regimens: (1) saline injections ( $2 \mathrm{ml} / \mathrm{kg}$, sc), (2) cocaine injections ( $40 \mathrm{mg} / \mathrm{kg}$ hydrochloride salt, sc, once a day, injected in $2 \mathrm{ml} / \mathrm{kg}$ volume), or (3) continuous cocaine infusion $(40 \mathrm{mg} / \mathrm{kg} /$ day, sc). Implantation and explantation procedures of osmotic minipumps (model 2ML2, Alza Corp., Palo Alto, CA) have been previously described (Joyner et al. 1993). Briefly, rats were anesthetized with methoxyflurane and a small incision was made on the back of skin (the incision site infiltrated with lidocaine), and the subcutaneous connective tissue was separated, forming a pocket. Single minipumps with modified flow modulators were inserted into this pocket and the wound closed with surgical clips. The pumps were removed 2 weeks later using a similar surgical procedure. For intermittent injections, the injection site was rotated daily throughout the 14-day period (in combination with the increased injection volume) to reduce skin inflammation associated with cocaine injection.

\section{Single-Unit Procedure}

Seven days after pretreatment withdrawal, rats were anesthetized with chloral hydrate $(400 \mathrm{mg} / \mathrm{kg}$, ip) and their jugular vein catheterized for drug administration (e.g., quinpirole). The animals were mounted on a Kopf stereotaxic apparatus. The skull was exposed, and a burr hole was drilled in the area overlying either the 
SNC or VTA, and the dura was removed. Body temperature of the rats was maintained at: 36 to $38^{\circ} \mathrm{C}$ using a temperature control pad.

Extracellular single-unit recordings were made using glass-coated tungsten electrodes (Frederick Haer, Brunswick, ME; impedance $10 \mathrm{M} \Omega$ at $1,000 \mathrm{~Hz}$ ). The number of spontaneously active SNC or VTA neurons exhibiting electrophysiological characteristics of DA neurons was counted by lowering the electrode from 6.5 to 8.5 mm below the brain surface (Chiodo and Bunney 1983). Nine electrode tracks, each separated by $0.2 \mathrm{~mm}$, were passed at predetermined coordinates $(3.1-3.5 \mathrm{~mm}$ anterior to lambda, $1.5-1.9$ and $0.4-0.8 \mathrm{~mm}$ lateral to the midline for SNC and VTA, respectively). The sequence of the electrode tracks was kept constant from animal to animal, and a new electrode was used for each animal. In addition to the number of spontaneously active neurons, the baseline firing rate and bursting pattern of each neuron were determined based on 500 consecutive spikes.

Putative DA neurons were identified by the previously established criteria: (1) a long action potential (>2.5 ms) with a distinct "notch" in the initial rising phase; (2) slow, burst, or regular firing pattern (0-9 spikes/s); and (3) low-pitched sound on an audio monitor. The bursting pattern of individual neurons was determined as described previously (Grace and Bunney 1984). A burst onset was defined as an occurrence of two spikes with an interspike interval of less than or equal to $80 \mathrm{~ms}$; an interval greater than $160 \mathrm{~ms}$ denoted a burst termination. For each neu:on, we determined: (1) average number of spikes per burst; (2) total number of bursts; and (3) \% of 500 collected spikes that occur within bursts.

When used, (-)-sulpiride (50 $\mathrm{mg} / \mathrm{kg}$, ip) was dissolved in $1 \%$ tartaric acid (normal saline) and injected $2 \mathrm{~h}$ before the start of the population sampling procedure. No differences between "naive" animals and those injected with acute vehicle were observed during the population sampling procedure; therefore, these two groups were combined for comparison to the sulpiride-injected group. For all animals, the experimenter was blind to the acute treatment given immediately before single-unit recording (i.e., no injection, saline, or sulpiride).

Dose-dependent inhibition of the SNC or VTA DA neurons by quinpirole was determined in separate groups of animals. After the isolation of a putative DA neuron, baseline data were collected for at least $5 \mathrm{~min}$. Subsequently increasing doses of the $\mathrm{D}_{2}$ agonist were administered via the juglar vein at 2-min intervals, until spontaneous activities ceased $(0.5-100 \mu \mathrm{g} / \mathrm{kg}$, iv, cumulative dose). Thirty minutes after the last dose, haloperi$\mathrm{dol}(50 \mu \mathrm{g} / \mathrm{kg}$, iv) was given to reverse the quinpiroleinduced impulse inhibition. Only one neuron per animal was used for quinpirole sensitivity determination.
At the end of the experiment, the last recording site was lesioned by passing $10-\mu \mathrm{A}$ current through the recording electrode for $30 \mathrm{~s}$. The animals were then perfused transcardially with $0.9 \%$ saline followed by $10 \%$ formalin under an overdose of chloral hydrate. Serial $50-\mu \mathrm{m}$ sections of the brain were stained with Cresyl violet for identifying the lesion. For the nine-track experiments, location of the recorded neurons were extrapolated based on that of the lesion site (i.e., the last recorded neuron).

\section{Drugs}

(-)-Sulpiride and haloperidol obtained from Sigma Chemical Co. (St. Louis, MO) was dissolved in $1 \%$ tartaric acid in saline immediately before injection. Quinpirole (RBI, Natick, MA) was dissolved in distilled water.

\section{Data Analysis}

Data acquisition and on-line analysis were accomplished using software written in Axobasic programming suite (Axon Instrument, Foster City, CA). Group differences in the number of spontaneously active neurons were assessed using a one-way analysis of variance (ANOVA; saline, intermittent injections, and continuous infusion, each group with or without sulpiride). For the baseline firing rates and bursting indices, a single set of values for each animal was first derived by averaging results from individual neurons; these values were subsequently used to compare pretreatment groups by a one-way ANOVA. Post hoc comparisons of individual means were accomplished using NewmanKeul's test.

The firing rate after each cumulative quinpirole dose was converted to the percentage of inhibition as the dependent variable and were evaluated by a two-way mixed model ANOVA (pretreatment $\times$ quinpirole dose). Multiple comparisons of individual means were accomplished with Dunnett's test. The quinpirole $\mathrm{ED}_{50}$ for each neuron was determined by the third-order polynomial regression fit of the log dose-response curve (Pitts et al. 1990). Differences of baseline firing rates and $\mathrm{ED}_{50}$ values were evaluated by one way ANOVA followed by Dunnett's test. For all tests, $p<$ .05 was considered statistically significant; all data are presented as mean $\pm \mathrm{SEM}$.

\section{RESULTS}

\section{Number of Active DA Neurons and Bursting Pattern}

Figure 1 presents representative extracellular recording traces for putative DA neurons sampled in the present study (see the legend for detail). These characteristics 


\section{ELECTROPHYSIOLOGICAL CRITERIA FOR PUTATIVE DOPAMINE NEURONS}

\section{Neuron without initial "notch"}

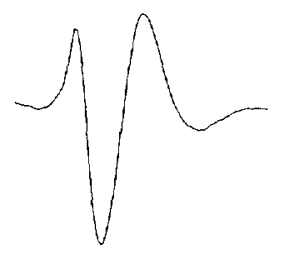

\author{
Neuron with initial "notch"
}

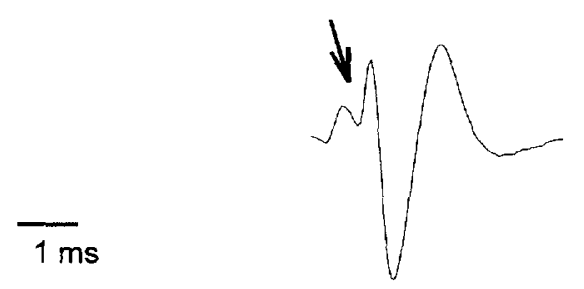

\section{Non-burster}

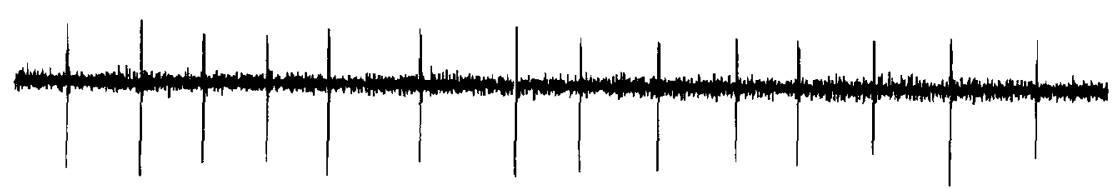

Burster

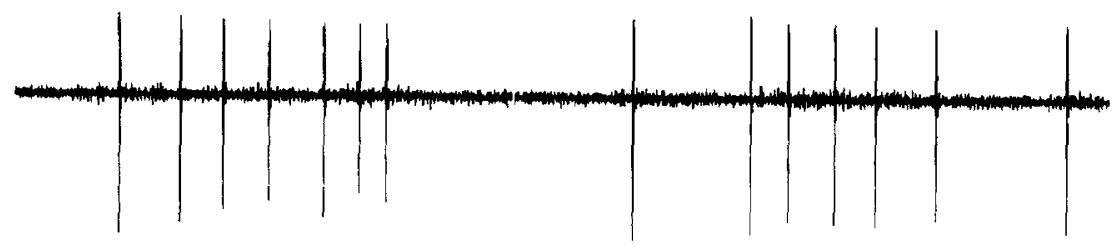

Firing rates: $\quad 4.9$ spikes/s Avg. \# sp./burst: 2

Tot. \# bursts: 1 $\%$ in bursts: $\quad 0.4$

Figure 1. Extracellular single-unit criteria used to identify putative DA neurons. Upper panel: These neurons exhibited long action potentials (>2.5 ms) with some neurons showing a pronounced "notch" (arrow) in the initial rising phase (compare the two neurons). Dopamine neurons were also identified by either regular (non-burster) or bursting (Burster) firing pattern (firing rates: $0-9$ spike/s). Furthermore, as shown in Figure 4, they were inhibited by low doses of $D_{2}$ agonist quinpirole, an effect readily reversible with haloperidol, a $D_{1} / D_{2}$ antagonist.

have been demonstrated previously to belong to DA neurons identified by l-dopa histofluorescence (Grace and Bunney 1980). All recording sites, as estimated from the location of the last recording sites, were localized between 2.96 and $3.70 \mathrm{~mm}$ anterior to the interaural line (Paxinos and Watson 1986). Correct medio-lateral positioning of the SNC and VTA sampling tracks were also verified histologically $(1.4-2.1 \mathrm{~mm}$ and $0.4-$ $1.2 \mathrm{~mm}$ lateral, respectively.

On day 7 of withdrawal from 14-day cocaine pretreatment, the six experimental groups (i.e., control, intermittent and continuous groups, with or without sulpiride) exhibited significant differences in the number of spontaneously active DA neurons in the SNC ( $p<$ .001 , one-way ANOVA; Figure 2). Before $D_{2}$ blockade with sulpiride, the group withdrawn from continuous cocaine infusion exhibited significantly reduced number of putative DA neurons compared to either the control or injection group ( $p<.05$, Newman-Keul's test). In contrast, the animals pretreated with intermittent injections exhibited a similar number of active DA neurons compared to controls.

Among various possibilities, two possible mechanisms underlying the reduced number of active DA neurons in the continuous group are induction of depolarization block (Chiodo and Bunney 1983) or autoreceptor supersensitivity (Bunney and Grace 1978; Zhang et al. 1992; Lee et al. 1993). To distinguish these two mechanisms, $\mathrm{D}_{2}$ antagonist sulpiride (50 $\mathrm{mg} / \mathrm{kg}$, ip) was administered $2 \mathrm{~h}$ before the sampling procedure. This antagonist was expected to exacerbate a reduced number of neurons if depolarization block was taking place. On the other hand, in case of autoreceptor supersensitivity, sulpiride would increase the number of spontaneously active DA neurons and "normalize" the number of active neurons in the continuous group by blocking the action of endogenous DA. Consistent with the autoreceptor hypothesis, the control, injection, and 
SNC

\# DA NEURONS/TRK.



AVG. \# SPIKES / BURST

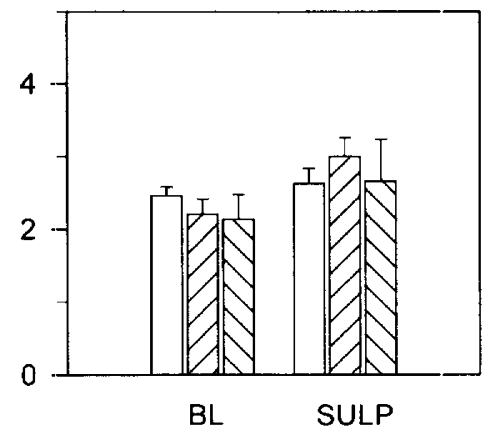

TOTAL \# OF BURSTS

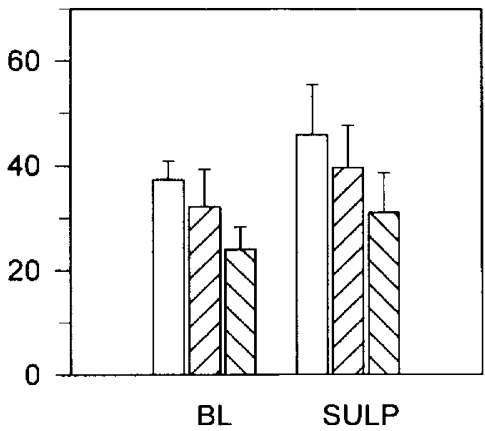

FIRING RATES ( ISEC)

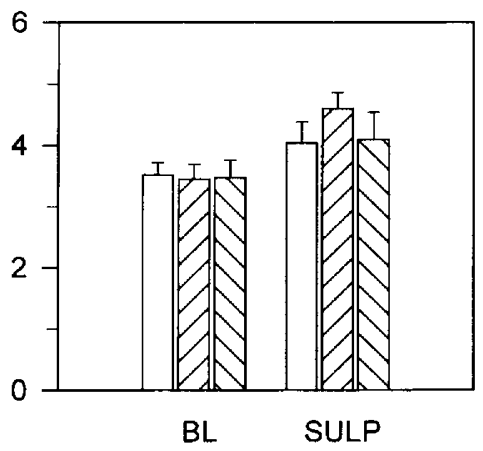

\% SPIKES IN BURSTS

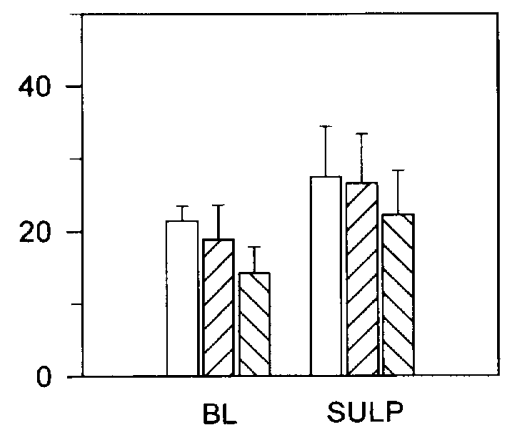

Figure 2. Effects of intermittent injections or continuous infusion on the baseline activity of single putative DA neurons in the SNC after 7-day withdrawal. For the baseline firing rates and bursting indices, results from individual neurons in each animal were combined to derive a single set of values. The data shown represent averages (mean \pm SEM) of these sets. INTI-intermittent injections, CONT-I-continuous infusion. BL-baseline condition with no acute drug injection, SULP-sulpiride, $50 \mathrm{mg} / \mathrm{kg}$, ip $2 \mathrm{~h}$ before the start of the "9-track" procedure. Number of animals: CONTROL, BL/SULP = 14/5; INT-I, BL/ SULP $=14.6$; CONT-I, BL/SULP $=10 / 7 .{ }^{*} p<.05$ compared with BL-CONTROL, Newman-Keul's test.

continuous groups all exhibited increased numbers of spontaneously active DA neurons following sulpiride $(p<.05$, Newman Keul's test, as compared to the respective baseline values: Figure 2). Most importantly, the number of active DA neurons in the continuous group was no longer different from either the control or injection groups. This finding is consistent with sulpiride blocking the supersensitive response to endogenous DA (also see below for quinpirole sensitivity).

In contrast to the number of active neurons, no significant differences in the baseline firing rates were observed among any of the pretreatment groups, with or without sulpiride ( $p>.07$, one-way ANOVA), although visual inspection suggested that sulpiride tended to increase firing rates in all pretreatment groups (Figure 2). Furthermore, neither intermittent nor continuous pretreatment regimen produced statisically significant re- sults on the bursting indices of putative SNC DA neurons (Figure 2).

In the VTA, the six experimental groups (i.e., control, intermittent, and continuous groups, with or without sulpiride) again exhibited significant changes in the number of active DA neurons after 7-day withdrawal $(p<.001$, one-way ANOVA; Figure 3). However, the pattern of group changes in this cell body region was strikingly different from the SNC. In this case, it was the intermittent injection group that exhibited an alteration, an increase ( $p<.05$, Newman-Keul's test). The number of VTA DA neurons in the continuous group did not differ from the control group. Overall, the baseline firing rates in the six groups were significantly different $(p<.02$, one-way ANOVA; Figure 3); however, comparisons of individual means failed to reach statistical significance. 


\section{VTA}

\# DA NEURONS/TRK.
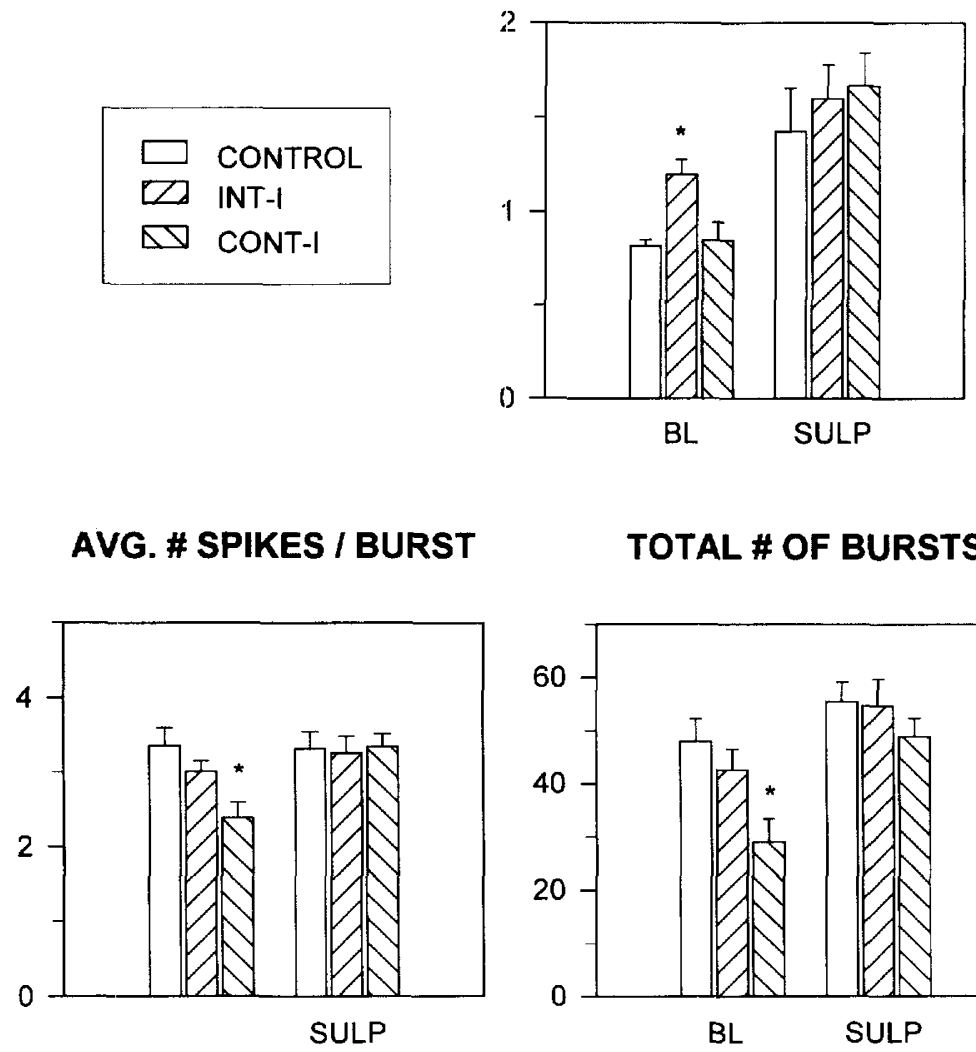

TOTAL \# OF BURSTS

AVG. \# SPIKES / BURST

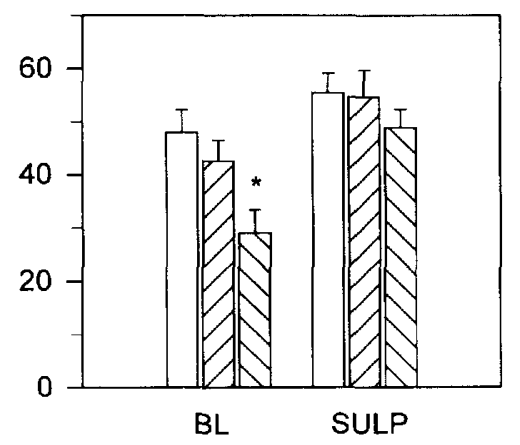

FIRING RATES ( / SEC)

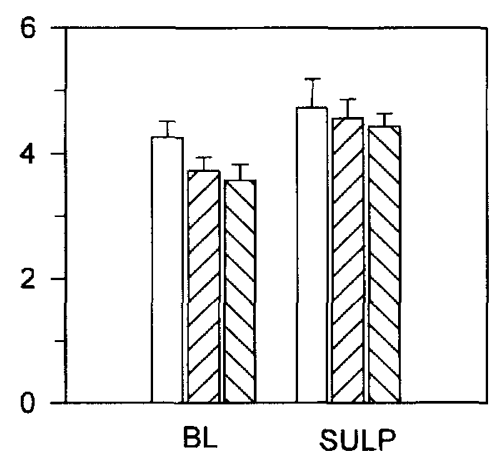

\section{\% SPIKES IN BURSTS}



Figure 3. Effects of intermittent injections or continuous infusion on the baseline activity of single putative DA neurons in the VTA after 7-day withdrawal. Results are presented as described in Figure 2 legend; see the same for group abbreviations. Number of animals: CONTROL, BL/SULP $=11 / 6$; INT-I, BL/SULP $=14 / 8 ;$ CONT-I, BL/SULP $=11 / 7 .{ }^{*} p<.05$, compared with BL-CONTROL, Newman-Keul's test.

The effects of sulpiride on the number of active DA neurons were also determined in the VTA. As in the $\mathrm{SNC}$, the $\mathrm{D}_{2}$ antagonist increased the number of DA neurons in all pretreatment groups compared to their respective no-sulpiride groups $(p<.05$, Newman Keul's test; Figure 3). Importantly, the intermittent group after sulpiride exhibited normal number of spontaneously active neurons, suggesting that the increased number of neurons under baseline conditions might be due to altered $\mathrm{D}_{2}$ response to endogenous DA.

Another significant set of findings in the VTA was altered baseline bursting activity after 7 -day withdrawal (Figure 3). Overall, significant group differences were observed for the total number of bursts $(p<.001), \%$ of total spikes firing in bursts $(p<.003)$ and average number of spikes per burst $(p<.009$, one-way ANOVA). Individual mean comparisons revealed that the infusion group exhibited significant reductions in all three indices $(p<.05$ vs. either control or injection group, New-
man-Keul's test). Interestingly, the decreased bursting in the VTA after 7-day withdrawal from continuous infusion was again not observable after sulpiride injection. The burst analysis, as in the SNC, distinguished the intermittent from continuous regimen groups in that the former group exhibited similar bursting activity compared with the controls, either before or after sulpiride (Figure 3).

\section{Dose-Dependent Inhibition by Quinpirole}

The sensitivity of single DA neurons to systemic quinpirole was measured to determine whether or not the altered number of spontaneously active DA neurons in either the intermittent or continuous group (or both) is associated with a corresponding change in $\mathrm{D}_{2}$ (autoreceptor) sensitivity (e.g., quinpirole supersensitivity and reduced number of spontaneously active neurons in the continuous group). Figure 4 presents representative 
SNC
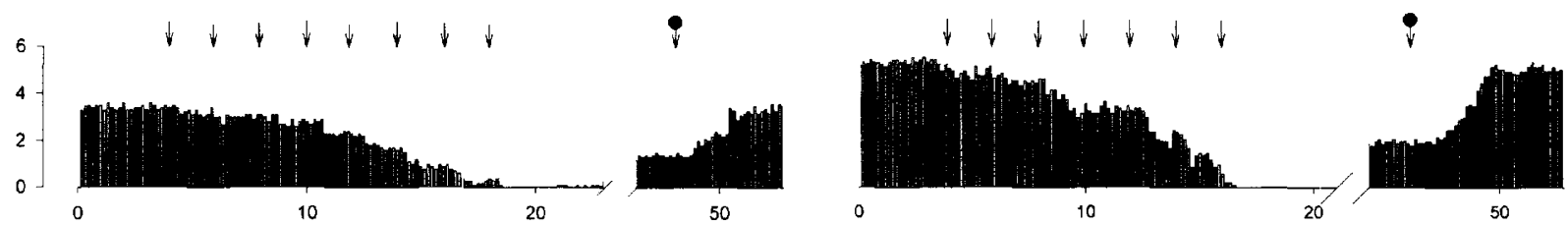

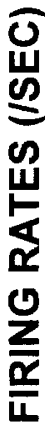
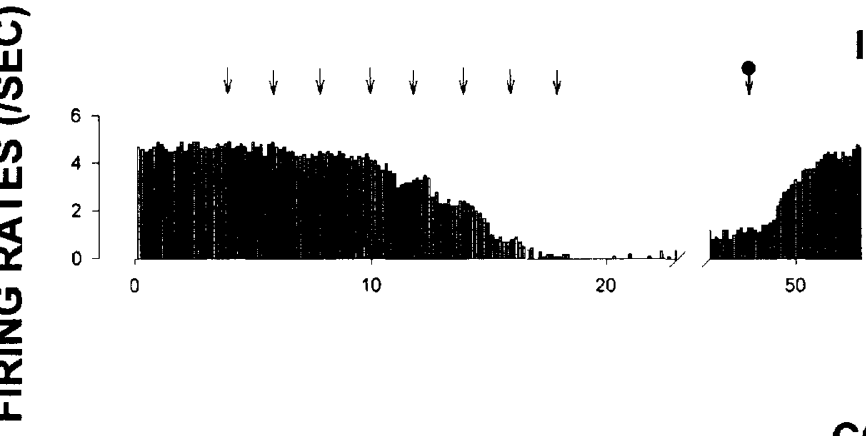

INT-I

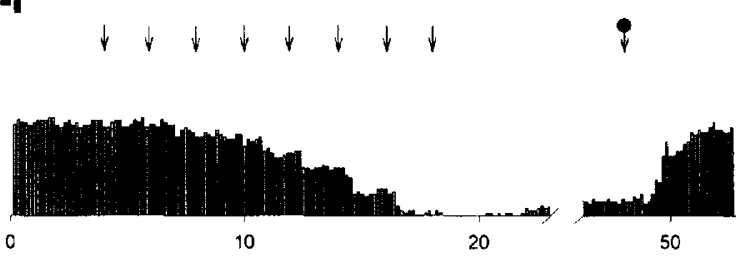

\section{CONT-I}
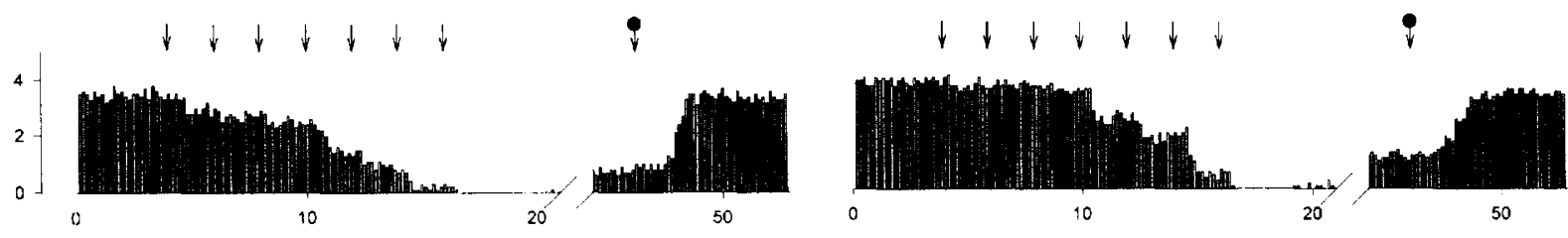

Figure 4. Representative cumulate rate histograms showing the inhibitory effects of quinpirole on single SNC and VTA DA neurons 7 days after withdrawal from intermittent or continuous cocaine administration. Arrows indicate the administration of quinpirole $(0.5,1,2,5,10,20,50,100 \mu \mathrm{g} / \mathrm{kg}$, iv, cumulative doses). Dotted arrows represent the beginning of haloperidol administration $(50 \mu \mathrm{g} / \mathrm{kg}$, iv). See Figure 2 legend for group abbreviations.

rate histograms showing dose-dependent inhibition of putative SNC and VTA DA neurons by quinpirole. In all pretreatment groups, complete inhibition of firing rates was achieved at $10-100 \mu \mathrm{g} / \mathrm{kg}$, iv, quinpirole; haloperidol $(50 \mu \mathrm{g} / \mathrm{kg}$, iv) given $30 \mathrm{~min}$ after the last quinpirole dose reversed the quinpirole-induce inhibition (85-105\% of baseline).

Overall, SNC DA neurons in the three pretreatment groups (i.e., control, intermittent injections, and continuous infusion) exhibited significantly different quinpirole sensitivity 7 days after withdrawal (Figures 4 and $5 ; p<.03$, two-way, mixed model ANOVA). Continuous cocaine administration for 14 days induced a significant increase in the quinpirole sensitivity as demonstrated by a shift of the dose-response curve to the left (Figure 5); individual dose comparisons were significant at cumulative doses of 5, 10, and $20 \mu \mathrm{g} / \mathrm{kg}(p<.05$, Dunnett's test). The quinpirole supersensitivity was also evident in decreased $\operatorname{ED}_{50}(10.2 \pm 1.4$, vs. $5.0 \pm 0.9$ $\mu \mathrm{g} / \mathrm{kg}$, iv, one-way ANOVA, $p<.02$, followed by Dunnett's test, $p<.05)$. The SNC DA neurons in the inter- mittent group showed no alteration in the quinpirole sensitivity $(10.0 \pm 1.2 \mu \mathrm{g} / \mathrm{kg}, \mathrm{iv})$.

In contrast to the SNC, DA neurons in the VTA exhibited similar sensitivity to quinpirole after withdrawal from either intermittent or continuous cocaine pretreatment (Figures 4 and 5). As in the population sampling experiments, no difference in the baseline firing rates was observed among groups in either the SNC or VTA (data not shown).

\section{DISCUSSION}

The present single-unit findings demonstrate that intermittent and continuous cocaine pretreatment regimens, which lead to differential behavioral and neurochemical outcome (Jones et al., 1996; Kilbey and Ellinwood 1977; King et al. 1992, 1994; Reith et al. 1987; Kalivas 1995; Weiss et al. 1995), also induce distinct changes in the baseline activity and quinpirole sensitivity of the SNC and VTA DA neurons. Thus, continuous cocaine 


\section{INHIBITION OF DOPAMINE NEURONS BY QUINPIROLE}



Figure 5. Effects of intermittent injections or continuous infusion on the quinpirole sensitivity of single DA neurons after 7-day withdrawal. Mean \pm SEM. See Figure 2 legend for group abbreviations. Only one neuron per animal was used (number of animals in parentheses). ${ }^{*} p<.05$, Dunnett's test.

infusion followed by 7-day withdrawal was associated with: (1) reduced number of spontaneously active neurons in the SNC; (2) decreased bursting in the VTA; and (3) selective quinpirole supersensitivity in the SNC. Intermittent injections, on the other hand, had minimal effects on the baseline activity of SNC DA neurons, while increasing the number of spontaneously active DA neurons in the VTA without altering the baseline bursting pattern or quinpirole sensitivity.

\section{Changes after Continuous Infusion}

The mechanism underlying the decreased number of SNC DA neurons after continuous cocaine infusion awaits further characterization. However, to the extent that altered soma/dendritic autoreceptor sensitivity might be associated with corresponding changes in the number of spontaneously active DA neurons (Bunney and Grace 1978; Henry et al. 1989; Harden and Grace 1995), the reduction might be mediated by supersensitivity of soma/dendritic autoreceptorsi. The present results with sulpiride and quinpirole $\left(\mathrm{D}_{2}\right.$ antagonist and agonist, respectively) are consistent with this hypothesis. Thus, the continuous group no longer exhibited a decreased number after an acute autoreceptor $\left(D_{2}\right)$ blockade with sulpiride and was supersensitive to the impulse-inhibiting effect of systemic quinpirole. We hypothesize that the latter effect is mediated via soma/ dendritic autoreceptors; we have previously demonstrated that continuous infusion of $\mathrm{d}$-amphetamine or cocaine induces, on day 7 of withdrawal, an enhanced sensitivity of single DA neurons to intravenous apomorphine or bath-applied DA (Ellinwood and Lee 1983; Lee and Ellinwood 1989; Lee et al. 1993; Zhang et al. 1992). In conclusion, evidence to date is consistent with the hypothesis that continuous infusion of cocaine (or amphetamine) leads to supersensitivity of soma/dendritic autoreceptors 7 days after withdrawal.

In addition to supporting soma/dendritic supersensitivity hypothesis, the sulpiride-induced normalization provides evidence against an induction of depolarization block for the decreased number of neurons. Such a block would have led to a further decrease in the number of active neurons (by removing hyperpolarizing influence of autoreceptors) or, at least, no change after sulpiride administration. In addition, the finding that the remaining active neurons in the infusion group were firing close to baseline firing rates and bursting 
patterns also argues against depolarization block. Neurons on the verge of entering depolarization block would likely exhibit increases in these indices.

Another striking finding after 7-day withdrawal from continuous cocaine infusion was decreased bursting activity in the putative VTA DA neurons. That this decrease is also not observed after acute sulpiride suggests an involvement of a $\mathrm{D}_{2}$-dependent mechanism. Because the soma/dendritic autoreceptor sensitivity in the VTA is apparently unaltered in the infusion group (normal number of spontaneously active DA neurons and normal quinpirole sensitivity), the $D_{2}$ mechanism(s) is likely to be localized outside the VTA. Among various possibilities, altered modulation of the excitatory input from the prefrontal cortex is an alternative. It has been shown that such input can modulate the bursting pattern of VTA or SNC DA neurons (Gariano and Groves 1988; Svensson and Tung 1989); enhanced DA neurotransmission in the prefrontal cortex (either increases in synaptic DA concentrations or DA receptor sensitivity) and a consequent decrease in the activity of the postsynaptic projection neurons (Deutch et al. 1990) may lead to a decreased bursting in the DA neurons. In this respect, it is interesting to note that intermittent cocaine injections, which induce opposite changes in a number of DA indices appear to lead to decreased DA neurotransmission in the prefrontal cortex (Kalivas 1995).

Burst firing in DA neurons may facilitate synaptic DA release in the terminal areas (Gonon 1988). Therefore, the reduced bursting in the: VTA DA neurons would lead to decreased basal level of DA in the nucleus accumbens after 7-day withdrawal from continuous infusion. In recent years it has been demonstrated that "unlimited" cocaine access under a self-administration paradigm (proposed to be analogous to continuous infusion) leads to a decrease in basal DA concentrations during the first few days of withdrawal (Weiss et al. 1995).

\section{Changes after Intermittent Injections}

In contrast to continuous infusion, intermittent cocaine injections did not alter the baseline activity or quinpirole sensitivity in the putative SINC DA neurons after 7-day withdrawal. Furthermore, acute sulpiride failed to induce changes that could distinguish the injection group from the other two (Figure 2). These results suggest that intermittent cocaine injections are unlikely to induce long-term (e.g., 7 days) changes in the sensitivity of SNC soma/dendritic DA autoreceptors.

In the VTA the intermittent regimen did lead to an increased number of spontaneously active DA neurons without an altered sensitivity to quinpirole. The normal number of active neurons after acute sulpiride injection suggests an involvement of $D_{2}$ receptors. However, the normal sensitivity to quinpirole suggests a mechanism other than subsensitive soma/dendritic autoreceptors; this conclusion is in agreement with previous reports (Henry et al. 1980; White et al. 1995).

One possible mechanism for the increased number of VTA neuron might be a change in postsynaptic $D_{2}$ receptors mediating the long-loop feedback inhibition. However, reports on changes in postsynaptic $D_{2}$ receptors after chronic cocaine/amphetamine injections have been conflicting (see Kalivas and Stewart 1991). In addition, White and his colleagues have reported that, after 7 days of withdrawal, the sensitivity of postsynaptic $D_{1}$ receptors in the nucelus accumbens is increased without accompanying $D_{2}$ changes (White et al. 1995). Another hypothesis that an altered responsivity of the VTA DA neurons to an extrinsic input (e.g., prefrontal cortex; Tong et al. 1995; White et al. 1995) mediates our current finding needs to be further evaluated, although such change would more likely affect the bursting activity of DA neurons (Gariano and Groves 1988; Svensson and Tung 1989). No changes in bursting were found in the injection group either in the SNC or VTA.

Ackerman and White (1992) have reported, after 10day withdrawal from their intermittent injection regimen, a decrease rather than an increase in the number of spontaneously active VTA DA neurons. Reasons behind the discrepancy between this previous report and our present results are not clear. Among possibilities, different doses and routes of pretreatment $(10 \mathrm{mg} / \mathrm{kg}$, ip twice a day vs. $40 \mathrm{mg} / \mathrm{kg}$, sc once a day) as well as the duration of withdrawal (10-14 days vs. 7 days) might account for the discrepancy. Further studies are needed to clarify these issues.

Previous studies have reported that soma/dendritic autoreceptor subsensitivity in the VTA is accompanied by increases in both the number of active DA neurons and the baseline firing rates in single DA neurons (e.g., Henry et al. 1989). In the present study, we did not find altered baseline firing rates in the putative SNC or VTA DA neurons after either continuous or intermittent regimens. Although it is well established that the responsivity of single DA neurons to autoreceptor stimulation correlates with their baseline firing rates under normal conditions (White and Wang 1984; Zhang et al. 1992; Pucak and Grace 1994; Lee et al. 1997), this index per se might not be a sensitive (reliable) indicator of altered soma/dendritic autoreceptor sensitivity after various experimental manipulations (e.g., 6-OHDA or cocaine pretreatment). Thus, we and others have reported altered neuronal sensitivity to direct DA agonists without corresponding changes in the baseline firing rates (Pucak and Grace 1991; Zhang et al. 1992; Harden and Grace 1995; White et al. 1995; Lee et al. 1997). Most recently, Tepper et al. (1997) have reported that inhibition of soma/dendritic autoreceptor synthesis by intranigral administration of $D_{2}$ antisense oligodeoxynucleotides 
alters the sensitivity of the SNC DA neurons to apomorphine without affecting the baseline firing rates. Being a composite of various presynaptic and postsynaptic influences, the baseline firing rates of DA neurons may reflect changes not only in autoreceptor sensitivity but also in other mechanisms (e.g., $\mathrm{D}_{1}$, sigma, or GABA; for review, see Grace and Bunney 1995) that were not specifically examined in the present study. Previously, Harden and Grace (1995) also suggested a similar explanation for a lack of correlation between changes in baseline firing rates/bursting and autoreceptor sensitivity after chronic l-DOPA treatment in DA-depleted animals.

In conclusion, the present study adds to the growing body of evidence that intermittent and continuous cocaine pretreatment leads to distinct alterations in various indices of central DA neurotransmission, including regulation of neuronal activity in the SNC and VTA (e.g., Henry et al. 1989; Lee et al. 1997; White et al. 1995; Zhang et al. 1992). Cocaine binge in humans is often characterized by simultaneous development of sensitization and tolerance to different effects of the drug (e.g., stereotyped thought pattern/paranoia and cocaine "high," respectively); furthermore, these "deficits" subside with differential time courses during abstinence/withdrawal (see Gawin et al. 1994 and Gold et al. 1992). Thus, when modeling changes associated with compulsive cocaine abuse and withdrawal, animal models describing either behavioral sensitization or tolerance without the other may not offer adequate experimental settings for full elucidation of critical mechanisms. Direct electrophysiological comparison and contrast of the two dosing regimens under identical experimental conditions may contribute to elucidation of alterations involved in chronic, compulsive cocaine abuse in hurnans.

\section{ACKNOWLEDGMENTS}

This work was supported by the National Institute on Drug Abuse (DA-06519 to T.H.L.). We thank Janes M. Tepper for helpful discussion and Zhiping Xiong for technical assistance.

\section{REFERENCES}

Ackerman JM, White FJ (1992): Decreased activity of rat A10 dopamine neurons following withdrawal from repeated cocaine. Eur J Pharmacol 218:171-173

Bunney BS, Grace AA (1978): Acute and chronic haloperidol treatment: Comparison of effects on nigral dopaminergic cell activity. Life Sci 23:1715-1727

Chiodo LA, Bunney BS (1983): Typical and atypical neuroleptics: Differential effects of chronic administration on the activity of A9 and A10 midbrain dopaminergic neurons. J Neurosci 3:1607-1619

Deutch AY, Clark WA, Roth RH (1990): Prefrontal cortical dopamine depletion enhances the responsiveness of mesolimbic dopamine neurons to stress. Grain Res 521:311-315

Ellinwood, Jr. EH, Lee TH (1983): Effect of continuous systematic infusion of d-amphetamine on the sensitivity of nigral dopamine cells to apomorphine inhibition of firing rate. Brain res 273:379-383

Emmett-Oglesby MW, Peltier RL, Depoortere CL (1993): Tolerance to self-administration of cocaine in rats: Time course and dose-response determination using a multidose method. Drug Alcohol Depend 32:247-256

Gallager DW, Pert A, Bunney WE Jr. (1978): Haloperidolinduced presynaptic dopamine supersensitivity is blocked by chronic lithium. Nature 273:309-312

Gariano RF, Groves PM (1988): Burst firing induced in midbrain dopamine neurons by stimulation of the medial prefrontal and anterior cingulate cortices. Brain Res 462:194-198

Gawin FH, Khalsa H, Ellinwood Jr. EH (1994): Stimulants. In Galanter M, Kleber HD (eds), Textbook of Substance Abuse. Washington, DC, American Psychiatric Press, pp 111-139

Gawin FH, Ellinwood Jr. EH (1988): Cocaine and other stimulants: Actions, abuse and treatments. N Engl J Med 318:1173-1182

Gold MS, Miller S, Jonas JN (1992): Cocaine (and Crack): Neurobiology. In Lowinson JH, Ruiz P, Millman RB, Langrod JG (eds), Substance Abuse: A Comprehensive Textbook. Baltimore, MD, Williams \& Wilkins, pp 222235

Gonon FG (1988): Nonlinear relationship between impulse flow and dopamine released by rat midbrain dopaminergic neurons as studied by in vivo electrochemistry. Neuroscience 24:19-28

Grace AA, Bunney BS (1980): Nigral dopamine neurons: Intracellular recording and identification with I-dopa injection and histofluorescence. Science 210:654-656

Grace AA, Bunney BS (1984): The control of firing pattern in nigra dopamine neurons: Burst firing. J Neurosci 4:2877-2890

Grace AA, Bunney BS (1995): Electrophysiological properties of midbrain dopamine neurons. In Bloom, FE, Kupfer, DJ (eds), Psychopharmacology: The Fourth Generation of Progress. New York, Raven Press, pp 163-177

Harden DG, Grace AA. (1995): Activation of dopamine cell firing by repeated L-DOPA administration to dopaminedepleted rats: Its potential role in mediating the therapeutic response to L-DOPA treatment. J Neurosci 15: 6157-6166

Henry DJ, Greene MA, White FJ (1989): Electrophysiological effect of cocaine in the mesoaccumbens dopamine system: Repeated administration. J Pharmacol Exp Ther 251:833-839

Jones SR, Lee TH, Wightman RM, Ellinwood EH (1996): Effects of intermittent and continuous cocaine administration on striatal dopamine neurotransmission following 7 day withdrawal: In vitro voltammetric assessment. Psychopharmacology 126:331-338

Joyner C, King GR, Lee TH, Ellinwood Jr. EH (1993): A technique for the continuous infusion of high doses of 
cocaine by osmotic minipump. Pharmacol Biochem Behav 44:971-973

Kalivas PW (1995): Neural basis of behavioral sensitization to cocaine. In Hammer Jr. RP (ed), The Neurobiology of Cocaine: Cellular and Molecular Mechanisms. Boca Raton, FL, CRC Press, pp 81-98

Kalivas PW, Stewart J (1991): Dopamine transmission in the initiation and expression of drug- and stress-induced sensitization of motor activity. Brain Res Rev 16:223-244

Kamata K, Rebec GV (1984): Nigral dopaminergic neurons: Differential sensitivity to apomorphine following longterm treatment with low and high doses of amphetamine. Brain Res 321:147-150

Kilbey MM, Ellinwood Jr EH (1977): Administration of stimulant drugs: Response modification. In Ellinwood, Jr EH, Kilbey MM (eds), Advances in Behavioral Biology: Cocaine and Other Stimulants. New York, Plenum Press, pp 409-429

King GR, Ellinwood Jr EH, Silvia C, Joyner CM, Xue Z, Caron MG, Lee TH (1994): Withdrawal from continuous or intermittent cocaine administration: Change in $D_{2}$ receptor function. J Pharmacol Exp Ther 269:743-749

King GR, Joyner C, Lee TH, Kuhn C, Ellinwood Jr EH (1992): Intermittent and continuous cocaine administration: Residual behavior states during vithdrawal. Pharmacol Biochem Behav 43:243-248

Lee TH, Ellinwood Jr EH (1989): Time-dependent changes in the sensitivity of dopamine neurons to low doses of apomorphine following amphetamine infusion. Brain Res 483:17-29

Lee $\mathrm{TH}$, Ellinwood EH, Zhang H (1993): In vitro extracellular recording from migra dopamine neurons following continuous d-amphetamine infusion. Eur J Pharmacol 232:125-129

Lee TH, Gao W-Y, Ellinwood EH (1997): Differential effects of SCH 23390 on the apomorphine subsensitivity in the substantia nigra and ventral tegmental area 1 day following withdrawal from contiouous or intermittent cocaine pretreatment. Brain Res 744:293-301

Paxinos G, Watson C (1986): The Rat Brain in Stereotaxic Coordinates, 2nd ed. San Diego, CA, Academic Press

Pitts DK, Freeman AS, Kelland MD, Chiodo LA (1989): Repeated amphetamine: Reduced dopamine neuronal responsiveness to apomorphine sut not quinpirole. Eur J Pharmacol 162:167-171

Pitts DK, Kelland MD, Freeman A.S, Chiodo LA (1993): Repeated amphetamine administration: Role of forebrain in reduced responsiveness of nigrostriatal dopamine neurons to dopamine agonists. J Pharmacol Exp Ther 264:616-621
Pitts SK, Kelland MD, Shen RY, Freeman AS, Chiodo LA (1990): Statistical analysis of dose-response curves in extracellular electrophysiological studies of single neurons. Synapse 5:281-293

Post RM, Contel NR (1983): Human and animal studies of cocaine: implications for development of behavior pathology. In Creese I (ed), Stimulants: Neurochemical, Behavioral, and Clinical Perspectives. New York, Raven Press, pp 169-203

Pucak ML, Grace AA (1991): Partial dopamine depletions result in an enhanced sensitivity of residual dopamine neurons to apomorphine. Synapse 9:144-155

Pucak ML, Grace AA (1994): Evidence that systemically administered dopamine antagonists activate dopamine neurons firing primarily by blockade of somatodendritic autoreceptors. J Pharmacol Exp Ther 271:11811192

Reith MEA, Benuck M, Lajtha A (1987): Cocaine disposition in the brain after continuous or intermittent treatment and locomotor stimulation in mice. J Pharmacol Exp Ther 243:281-287

Svensson TH, Tung C-S (1989): Local cooling of prefrontal cortex induces pacemaker-like firing of dopamine neurons in rat ventral tegmental area in vivo. Acta Physiol Scand 136:135-136

Tepper JM, Sun B-C, Martin LP, Creese I (1997): Functional roles of dopamine $D_{2}$ and $D_{3}$ autoreceptors on nigrostriatal neurons analyzed by antisense knockdown in vivo. J Neurosci 17:2519-2530

Tong Z-Y, Overton PG, Clark D (1995): Chronic administration of $(+)$-amphetamine alters the reactivity of midbrain dopaminergic neurons to prefrontal cortex stimulation in the rat. Brain Res 674:63-74

Weiss F, Parsons LH, Markou A (1995): Neurochemistry of cocaine withdrawal. In Hammer Jr RP (ed), The Neurobiology of Cocaine: Cellular and Molecular Mechanisms. Boca Raton, FL, CRC Press, pp 163-181

White FJ, Hu X-T, Henry DJ, Zhang X-F (1995): Neurophysiological alterations in the mesocorticolimbic dopamine system with repeated cocaine administration. In Hammer Jr RP (ed), The Neurobiology of Cocaine: Cellular and Molecular Mechanisms. Boca Raton, FL, CRC Press, pp 99-119

White FJ, Wang RY (1984): A10 dopamine neurons: Role of autoreceptors in determining firing rate and sensitivity to dopamine agonists. Life Sci 34:1161-1170

Zhang H, Lee TH, Ellinwood Jr. EH (1992): The progressive changes of neuronal activities of the nigral dopaminergic neurons upon withdrawal from continuous infusion of cocaine. Brain Res 594:315-318 